\title{
La troisième livraison de l'Atlas de la Suisse
}

Georges Lobsiger

La troisième livraison de l'Atlas de la Suisse, soit 10 cartes, ne le cède en rien aux deux précédentes. $\mathrm{La}$ rigueur scientifique s'y associe une fois encore à l'élégance de l'exécution des cartes et des diagrammes. Ce monument cartographique et ses commentaires, sobres et savants, font honneur aux rédacteurs et aux dessinateurs. On a parlé antérieurement du choix heureux des échelles au $1: 500000^{\mathrm{e}}$ et $1: 800000^{\mathrm{e}}$ pour les cartes principales.

La carte $\mathrm{N}^{\circ} 12$, appuyée sur des diagrammes révélateurs, matérialise les effets du relief sur les précipitations et permet de comprendre la difficulté du repérage exact des isohiètes dans les régions accidentées. Elle s'associe intimément aux cartes déjà parues consacrées aux terres ouvertes, à la couverture végétale, à la production agricole, tant sur le plan horizontal que vertical.

A côté des limites classiques des bassins hydrographiques ou fermés, la carte $\mathrm{N}^{\circ} 14$, dédiée à l'hydrologie générale y compris les glaciers, les lacs artificiels et les aqueducs alpins en galerie, situe encore les travaux de protection des avalanches et les stations d'épuration des eaux usées. L'échelle choisie permet ce luxe de détails qui ne surchargent pas la carte.

Les potamologues s'attarderont sur les diagrammes de la carte $\mathrm{N}^{\circ} 15$ et les hydrologistes verront dans les deux cartons au $1: 25000^{\mathrm{e}}$ consacrés au régime hydrographique dans une région aux roches imperméables et une autre fort perméable, une excellente introduction à la carte $\mathrm{N}^{\circ} 16$, vouée à l'hydrogéologie qui s'inscrit dans le programme de la décennie hydrologique mondiale. Il est bon de comparer cette carte avec celle au 1:1000 000 des nappes d'eau souterraines de France et à celle au 1:100 000 de la Thurgovie (1952) qui ne mentionnent que les régions aquifères, sans s'occuper de la géologie superficielle. Une conception féconde a guidé le rédacteur de cette carte remarquable qui montre non seulement les emplacements des terrains susceptibles d'être des réservoirs en profondeur mais encore les régions stériles. Les zones géologiques à forte ou faible pérméabilité sont délimitées avec soin, compte tenu de la nuance entre les calcaires alpins, qui contiennent plus de couches imperméables que ceux du Jura, la région aride par excellence. Les zones préférentielles de peuplement, de travail et de communication, autrement dit les vallées du bassin de l'Aar dans le Moyen-Pays, contiennent toutes des graviers et des alluvions aquifères. On peut espérer qu'une prospection méthodique décèlera assez de nappes souterraines pour soulager les autorités et les hygiénistes. La précision de la notation est telle que des mini-plaines alluviales d'altitude, comme dans la vallée de Saas, figurent à côté des grandes régions hydrologiques.

La mutation des classes d'âge entre 1910, 1941 et 1960 apparaît dans les diagrammes de la carte $\mathrm{N}^{\circ} 25$, intitulée «Etat et mouvement de la population». De la pyramide régulière de 1910 , on passe au profil "en meule de foin» de 1941 pour arriver en 1960, pour les seuls Suisses, au type du clocher bulbeux, avec un étonnant remplissage dû à la présence d'une importante colonie de jeunes adultes étrangers (20 à 40 ans). Le coup de frein donné par ces immigrants laborieux au vieillissement de la population est visible à travers ces diagrammes révélateurs.

La carte $\mathrm{N}^{\circ} 28$, consacrée aux dialectes et à la prononciation locale de mots choisis, introduit le lecteur dans un monde plus fragmenté que la carte générale des langues $\left(\mathrm{N}^{\circ} 27\right)$ ne le laissait supposer. Les cartes $\mathrm{N}^{\circ} 73$ et 74 , qui matérialisent les services aériens suisses et l'activité des PTT sont pour ainsi dire une préface à la carte $\mathrm{N}^{\circ} 64$ qui s'attache aux problèmes du tourisme et à la figuration schématique et précise de la fréquentation des hôtels par la clientèle suisse et étrangère, avec la discrimination entre lieux de transit et lieux de séjour, d'après les périodes sportives et selon une forte décentralisation spatiale et altimétrique.

Tout esprit porté à la méditation politique s'arrêtera longuement sur la carte $\mathrm{N}^{\circ} 33$ qui présente sous une forme singulièrement expressive les migrations alternantes quotidiennes. Les communes d'appel aux travailleurs du dehors, les communes d'émigration de salariés, les communes équilibrées entre ces deux tendances et celles où la population laborieuse se confond avec celle de résidence, traduisent la mutation profonde causée en Suisse par la conjoncture qui a accentué le régime antérieur des communes-dortoirs. Malgré l'égalité théorique des communes, une vingtaine de celles-ci occupent une position de commandement à l'intérieur d'ensembles de communes-réservoirs de main-d'œuvre en salopette ou en col blanc. Les itinéraires peuvent être courts. D'autres atteignent 60 kilomètres. La puis- 
sance d'attraction de ces communes directrices sur les communes salariées, si l'on peut s'exprimer ainsi, déséquilibre la notion classique de la commune, non seulement celle de la commune d'origine dans laquelle naissent et vivent de moins en moins les Suisses, mais encore celle de la commune de domicile ou commune politique, au profit de la commune professionnelle. C'est une des causes du malaise actuel, car la règle ne coïncide plus exactement avec le comportement, la situation de droit ne recouvrant plus totalement la situation de fait. Il sera facile d'annuler ce hiatus en aménageant, sans les meurtrir, des pratiques qui ont fait leurs preuves et qui pourront s'adapter aux nouvelles situations créées par la mutation économique et la répartition actuelle de la population. On comparera avec fruit cette carte à celle de W. Stammherr $\left(\mathrm{N}^{\circ} 31\right)$ qui montre la modification économique et démographique subie par la Suisse entre 1910 et 1960.

L'analyse spectrale de la Suisse matérialisée par
l'Atlas de la Suisse a déjà fourni de remarquables images de la réalité suisse, images qui doivent remplacer les stéréotypes périmés et les représentations collectives désuètes. Cette carte $\mathrm{N}^{\circ} 33$ mérite un examen minutieux, car elle montre la face nouvelle des relations du travail et du domicile, en même temps qu'elle fait mieux comprendre l'érosion du temps libéré par la longueur des déplacements quotidiens. Elle présente avec vigueur le dynamisme de quelques villes qui savent utiliser les habitants de leur voisinage immédiat et de leur arrière-pays à l'édification de leur prospérité, enviable et méritée. Cet atlas mérite son qualificatif de national. Il l'est pour le simple citoyen qui veut connaître le pays sous son aspect actuel et non selon des souvenirs scolaires. Il le sera absolument lorsqu'il sera devenu la source de documentation et de réflexion des autorités et des parlementaires, au niveau cantonal comme à l'échelon fédéral. Il suggère des réponses suisses aux problèmes suisses. 